\section{Evaluation of Canalization of the Canaliculus with Digital Photo System for Treating Dry Eye}

Purpose: We developed a simple evaluation technique of canalization of the canaliculus by observing sequential images of the meniscus height after instillation of fluorescein solution.

Subjects and Methods: We used nine eyes of eight patients [one male, seven female, age: $68.3 \pm 15.9$ (mean \pm standard deviation)] whose punctal plugs were extruded due to granulation formation within the canaliculi. Fluorescein solution was instilled into the eyes, until the tear meniscus was of sufficient height. Then the tear meniscus at the center of the lower lid margin was photographed with a slit-lamp equipped with a digital camera every three minutes for 15 minutes. These sequential photographs were displayed on the screen of a personal computer and from these photographs, canalization of the canaliculus was evaluated.

Results: In six eyes of six subjects, the meniscus height was remarkably diminished with time and the meniscus showed significant decrease as late as 15 minutes. In three eyes of two subjects, no remarkable decrease was noted during the 15 minutes.

Conclusion: This simple method can be usefully applied in a clinical situation, because canalization of the canaliculus is easily evaluated just by looking at the sequential meniscus images on the computer display.

Nippon Ganka Gakkai Zasshi (J Jpn Ophthalmol Soc 107:526-529, 2003)

Kunio Maruyama, Norihiko Yokoi, Masakazu Nishii and Shigeru Kinoshita

Department of Ophthalmology, Kyoto Prefectural University of Medicine

DOI 10.1007/s10384-004-1012-8

\section{Effect of Latanoprost on Diurnal Variations of Intraocular Pressure in Normal-tension Glaucoma}

Objectives: To study the effect of instillation of latanoprost on the diurnal variation in the intraocular pressure (IOP), blood pressure, and pulse rate in patients with normaltension glaucoma (NTG).

Subjects and Methods: The diurnal variation in the IOP was determined in 23 eyes of 23 NTG patients after a washout period of 4 weeks or longer. The diurnal variation in IOP was then remeasured after latanoprost monotherapy of 8 weeks or longer. The IOP was measured by the Goldmann applanation tonometer at 10:00, 13:00, 16:00, 19:00, 22:00, 01:00, 03:00, 07:00, and 10:00 before and after treatment, and the IOP at each time point, mean diurnal IOP, maximum IOP, minimum IOP, and range of variation in IOP were compared before and after treatment. The blood pressure and pulse rate before and after treatment were also measured and compared.

Results: The IOP decreased significantly at all time points. The treatment caused significant decreases in the mean diurnal IOP, maximum IOP, minimum IOP, and range of variation in IOP. There were no differences in the blood pressure and pulse rate before and after treatment.

Conclusions: Latanoprost significantly decreases the IOP throughout the day in NTG patients, and has no effects on the blood pressure and pulse rate. It is therefore useful in the treatment of NTG.

Nippon Ganka Gakkai Zasshi (J Jpn Ophthalmol Soc 107:530-534, 2003)

Kenji Nakamoto, Noriko Yasuda, Mami Nanno, Yayoi Kinohira, Keiko Murai and Takumi Fukuda

Department of Ophthalmology, Tokyo Metropolitan Police

Hospital

DOI 10.1007/s10384-004-1013-7

\section{A Case of Endogenous Aeromonas hydrophila Endophthalmitis}

Background: Aeromonas hydrophila (A. hydrophila) may cause septicemia in immunocompromised hosts, but endophthalmitis due to this agent is quite rare. To our knowledge, there have been no reports of $A$. hydrophila endophthalmitis in Japan. We report a case of endogenous endophthalmitis caused by Aeromonas hydrophila.

Case: The patient was a previously healthy 71-year-old man and no source of the endogenous endophthalmitis was found. A diagnostic/therapeutic vitrectomy was performed, but it was impossible to successfully treat this case because of extended retinal necrosis. A. hydrophila was isolated from the vitreous specimen.

Conclusion: Endophthalmitis due to A. hydrophila had a rapid clinical course and poor prognosis.

Nippon Ganka Gakkai Zasshi (J Jpn Ophthalmol Soc 107:535-537, 2003)

Tomonori Tamura and Tetsuo Hida

Department of Ophthalmology, Kyorin University School of Medicine

DOI 10.1007/s10384-004-1014-6

\section{Herpes Simplex Keratitis after Ophthalmic Surgery}

Purpose: We report 6 cases of herpes simplex keratitis after ophthalmic surgery, in eyes without clinical history of herpes simplex keratitis. 